\title{
thumanidades
}

Revista Humanidades

ISSN: 2215-3934

humanidades@ucr.ac.cr

Universidad de Costa Rica

Costa Rica

\section{Sobre la maleabilidad de las creencias de la comunidad científica}

Bermúdez Vargas, Br. Juan José; Castillo Rodríguez, Dr. Rodrigo

Sobre la maleabilidad de las creencias de la comunidad científica

Revista Humanidades, vol. 11, núm. 2, 2021

Universidad de Costa Rica, Costa Rica

Disponible en: https://www.redalyc.org/articulo.oa?id=498066660002

DOl: https://doi.org/10.15517/h.v11i2.47316

\section{(c) (1) $\Theta \Theta$}

Esta obra está bajo una Licencia Creative Commons Atribución-NoComercial-SinDerivar 3.0 Internacional. 
Desde las ciencias exactas, la tecnología y el conocimiento

\title{
Sobre la maleabilidad de las creencias de la comunidad científica
}

About the Malleability of Beliefs in the Scientific Community

Br. Juan José Bermúdez Vargas

Escuela de Física y Centro de Investigaciones Geofísicas,

Universidad de Costa Rica, San José, Costa Rica

DOI: https://doi.org/10.15517/h.v11i2.47316

Redalyc: https://www.redalyc.org/articulo.oa?

juanjose.bermudez@ucr.ac.cr

(iD https://orcid.org/0000-0002-0641-9633

Dr. Rodrigo Castillo Rodriguez

Escuela de Física y Centro de Investigaciones Geofísicas,

Universidad de Costa Rica, San José, Costa Rica

rodrigo.castillorodriguez@ucr.ac.cr

(iD https://orcid.org/0000-0003-0866-7435

Recepción: 07 Noviembre 2020

Aprobación: 11 Abril 2021

\section{ReSUMEN:}

Se propone estudiar la influencia de las condiciones externas más cercanas a una comunidad científica sobre las creencias personales a las que se tienden a acoger los individuos que forman parte de esta comunidad. Para esto se realiza un recorrido histórico, primero, partiendo desde el origen de las religiones en las civilizaciones más antiguas, segundo, comentando el impacto del determinismo impulsado por la mecánica newtoniana y, finalmente, advirtiendo un retorno al misticismo provocado por la falta de una interpretación completa de la mecánica cuántica. Se concluye que existe una correlación significativa entre el estado de la ciencia en un periodo en particular y las creencias que mantienen los individuos de la comunidad científica asociada a tal periodo.

Palabras Clave: ciencia, religión, misticismo, ateísmo.

\begin{abstract}
:
The aim of this essay is to study the influence of external conditions close to a scientific community over the personal beliefs that those who are part of it tend to hold. A historical approach is given on the subject, first, starting from the origin of religions in ancient civilizations, second, commenting the impact of determinism driven by Newtonian mechanics, and, finally, highlighting a return to mysticism caused by the lack of a complete interpretation of quantum mechanics. It is concluded that there exists a significant correlation between the state of science in a particular time period and the beliefs held by the members of the scientific community associated with that period.
\end{abstract}

KEYWORDS: science, religion, mysticism, atheism.

\section{INTRODUCCIÓN}

Dentro de la comunidad científica asociada a las ciencias naturales, existe actualmente una noción de desconexión completa de sus campos de estudio con respecto a las humanidades. Este ensayo busca desafiar esta idea, equivocada, desde nuestro punto de vista como físicos (y, por ende, miembros de tal comunidad científica). Se busca demostrar la íntima relación entre ambas áreas. Asimismo, se propone un modelo a través del cual tanto las ciencias naturales como la comunidad humana que practica las humanidades se desarrollan de la mano, influenciándose mutuamente en un bucle de retroalimentación constante. El campo de estudio motiva una serie de cambios en la forma de pensar del individuo investigador y cómo este percibe al mundo que lo rodea, lo cual a su vez modifica la forma en que tal individuo reacciona a los problemas de su campo y cómo los aborda. De esta forma impacta el desarrollo del campo y, por tanto, el campo mismo. Finalmente, 
por brevedad, se utilizará en adelante la palabra ciencia para referirse específicamente al campo de las ciencias naturales.

Para iniciar esta discusión, basta recordar un punto clave que caracteriza a la comunidad científica; en su sentido más básico, es una agrupación humana con intereses comunes. Vista de esta forma, resulta imposible pensar que una comunidad científica y, por lo tanto, la ciencia que esta desarrolla, carezca por completo de una realidad humana subyacente que interfiera e impacte su desarrollo. Todo ser humano lleva consigo una serie de creencias, contenidos de lo inconsciente colectivo denominados arquetipos. Los arquetipos definen su comportamiento moral a la vez que modifican su visión del mundo y cómo interactúa con este (Jung, 1954). Entonces, emerge un interesante cuestionamiento: ¿las condiciones del entorno en el que se desarrolla una persona poseen algún efecto sobre las creencias a las que decide asociarse dicha persona? Se encuentra aquí la tesis principal de este ensayo. Se busca demostrar cómo las condiciones que rodean, en particular, a la comunidad científica poseen un efecto directo sobre el grado de creencia en elementos espirituales que vayan a mantener sus miembros (ya sea esta una tendencia hacia el teísmo o hacia el ateísmo, en comparación con la población general). Es importante aclarar que las condiciones referidas aquí son aquellas que describen directamente el estado de la ciencia en cierto momento determinado. Se dejarán de lado las condiciones que no sean específicamente de esta índole (una categoría que se volverá más clara a medida que se avanza con la lectura del texto).

Antes de efectuar un proceso de demostración, cabe destacar que el punto que se abarca no supone una conclusión totalmente determinista, puesto que la mente humana es un órgano que esconde infinitas complejidades. La tesis a la que apunta este ensayo deberá interpretarse entonces de una forma aproximada, como una tendencia hacia el teísmo o el ateísmo, sin pretender una completa homogeneidad en relación con la comunidad científica.

Este ensayo recurrirá a tres distintos argumentos para arribar, de modo lógico, a la conclusión de que las condiciones científicas impactan directamente las creencias de la comunidad científica. Debido a que este es un tema afín a la mente humana, se iniciará con la referencia al campo de la psicología.

Como segundo argumento, se realizará un recorrido breve por la historia de la ciencia previa al auge de la mecánica cuántica. En esta parte, se enfatizará en puntos clave, los cuales fueron seleccionados por su importancia en el desarrollo de los grandes esquemas de la ciencia moderna y, a la vez, por la claridad con la que estos se acoplan al objeto de este ensayo para corroborar su tesis. Finalmente, se establece un tercer argumento vinculado con el presente científico y se emplea el nacimiento de la mecánica cuántica como punto de partida. Este último argumento conlleva el estudio de un fenómeno de maleabilidad de creencias que aún se encuentra vivo y en pleno desarrollo dentro de la comunidad científica. Hasta este punto se habrá comprendido cómo este (el tercer argumento) es una clara consecuencia de la idea principal del ensayo. Con fortuna, la persona lectora habrá adquirido un enfoque nuevo sobre la ciencia, así como su proceso de producción. En otras palabras, se habrá adquirido una perspectiva de utilidad tanto para las personas colegas de la comunidad científica, quienes hallarán un nuevo sentido de conexión con las humanidades, como para quienes realizan estudios en el área de las humanidades, quienes habrán podido profundizar su entendimiento en cuanto a la dinámica que domina a la comunidad científica desde el punto de vista de sus integrantes.

\section{LA HIPÓTESIS DEL REEMPLAZO DE CREENCIAS}

Tal como se adelantó en la introducción, este primer argumento se centra en observaciones de la psicología. En este sentido, la psicología del ateísmo ha sido ya tema de múltiples estudios elaborados en el área de las ciencias sociales. Debido a lo anterior, se tomará esta disciplina como punto de partida para esta discusión. Farias (2013) concluyó, a partir de varios estudios psicológicos, que la persona religiosa es, generalmente, menos hábil que su contraparte atea en tareas que requieren un razonamiento analítico. 
Ahora bien, la ciencia fundamenta que su progreso se debe al pensamiento crítico y lógico, por lo que se le reconoce como el epítome del razonamiento analítico. De esta manera, los miembros de la comunidad científica, inmersos en el entorno que esta genera (y exige), terminan adaptándose y volviéndose especialistas en esta forma de pensar. Hasta aquí, se concluye el primer indicio hacia la tesis principal de este ensayo: la comunidad científica experimenta, posiblemente, una mayor susceptibilidad a dejar de lado el teísmo, producto de (o correlacionado con) su pensamiento analítico entrenado. Esta susceptibilidad estaría relacionada con una relajación de las restricciones intrínsecas del individuo, las cuales evitan que él o ella abandone las creencias que ha mantenido, quizá, durante gran parte de su vida. Esta última afirmación posiblemente les recordará, a algunas de las personas de la comunidad científica, una de las tantas facetas de la ciencia que cualquier investigador o investigadora conoce bien: la ciencia es un campo en continuo estado de cambio. En este sentido, para seguirle la pista hay que estar anuente a lidiar con estas modificaciones y adaptarse a ellas, si es que se pretende contribuir con el desarrollo del campo. De esta forma, no es sorpresivo el hecho de que los miembros de la comunidad científica experimenten tal relajación de sus restricciones intrínsecas. Incluso, se podría decir que han recibido un entrenamiento indirecto para contrarrestar estas últimas.

Para profundizar aún más en la psicología de la persona atea, Farias (2013) aborda en su trabajo la hipótesis del reemplazo de creencias. Esta hipótesis sugiere que el ser humano, por naturaleza, busca algo en qué creer para hallar un apoyo emocional en medio de situaciones adversas. Para la mayoría de las personas, la religión cumple este papel, sin embargo, esta no es una función exclusivamente reservada a ella. Ahora bien, si la hipótesis pretende ser correcta, debería existir una fuente de apoyo a la que las personas ateas acudan ante la ausencia de figuras sobrenaturales o arquetípicas. Una primera sugerencia que Farias (2013) introduce en su trabajo para solventar esta problemática proviene del psicólogo John Gray (2004), quien opina que las personas ateas tienden a encontrar una fuente de apoyo en la idea del progreso humano. Esta idea luego sería corroborada científicamente por Rutjens et al. (como se citó en Farias, 2013).

A partir de lo anterior, una observación rápida podría sugerir que la comunidad científica presenta esta misma creencia en el progreso de la humanidad, específicamente, a través del avance en la ciencia. La ciencia es, según lo expuesto hasta este momento, una empresa interminable. Esta ha excedido la vida de todos aquellos que se dedicaron a ella en el pasado y, muy probablemente, excederá la de todos aquellos que leen ahora mismo estas palabras. Si aún se aferra el ser humano a esta empresa perdida, seguramente será porque confía en que su trabajo, aunque esté destinado a la incompletitud, añadirá algo a la colección de herramientas disponibles para las generaciones futuras en su continuación de la empresa científica. Así, sin darse cuenta se ha dotado a la comunidad científica de cualidades heroicas que, lastimosamente, no siempre están presentes. Como menciona el mismo Farias (2013) en su lectura, la adscripción a la ciencia no exige, de ningún modo, una fe en la evolución positiva de la sociedad.

Si bien practicar la ciencia no exige esta fe en el progreso humano, el trabajo de Farias (2013) identifica de forma independiente la creencia en la ciencia como posible fuente de apoyo para el ser humano en situaciones adversas. La ciencia representa una forma sistemática de entender el mundo sin recurrir a explicaciones de carácter sobrenatural o arquetípico. Para comprobar esto fueron diseñados dos experimentos con el fin de estudiar la respuesta de individuos en situaciones de alto estrés y sus respectivos grados de creencia en la ciencia como fuente de conocimiento. Los individuos expuestos a estos estados de tensión reportaron en ambos experimentos un mayor grado de confianza en la ciencia respecto de los grupos de control (Farias et al., 2013). Si bien esto no comprueba que la creencia en la ciencia lleve a una disminución de los niveles de estrés, sí sugiere la posición de que la ciencia actúe como posible mecanismo para lidiar con situaciones adversas.

Este análisis dará por sentado dos resultados como ciertos, los cuales, según se ha visto, están apoyados en evidencia experimental. En primer lugar, la hipótesis del reemplazo de creencias se cumple. En segundo lugar, la confianza en la ciencia como fuente de conocimiento puede funcionar como fuente de seguridad en situaciones adversas. Asumiendo estos dos enunciados, resulta natural que la persona que pertenece al campo 
de la ciencia se vea menos inclinada hacia la religiosidad, puesto que sus necesidades ya se ven parcialmente satisfechas por otros medios. Cabe mencionar que la decisión de aceptar el dogma científico es una apuesta que parte desde el individuo, como sucede con cualquier religión. El grado en que una persona está dispuesta a aceptar este dogma científico dependerá, como sucede en los juegos de cartas, del juicio de la persona, tras haber evaluado sus probabilidades de ganar -o bien, de no haberse equivocado-. Esta afirmación no busca reducir el proceso de adscripción a un dogma al equivalente de un juego de cara o cruz, sino explicar la naturaleza de la situación a la que se enfrenta un individuo cuando se ve obligado a escoger. La decisión final de tal individuo será, ultimadamente, determinada por una multitud de factores que los autores no traen a colación en este momento, pero que igualmente condicionan la forma en la que una persona actúa dentro de los procesos de toma de decisiones. Se concluye así que tanto el entorno científico como las posibilidades que este le ofrezca a cada individuo son posiblemente los factores más relevantes dentro del proceso de inclinación más pronunciada o moderada hacia el ateísmo en una comunidad científica.

Es, en este punto de la discusión, cuando la disciplina de la historia se vuelve útil, en cuanto herramienta para explorar esta relación entre el entorno y las creencias del ser humano que pertenece al campo de la ciencia. No obstante, la historia humana no ha sido exactamente corta; aun circunscribiéndose únicamente a la historia de la ciencia, resulta un tema vasto. Por esta razón, se han de visitar tan solo determinados puntos de interés. Como se explicó anteriormente, se seleccionaron para este efecto puntos clave dentro de la historia del desarrollo de la ciencia moderna. Siempre existirá, indudablemente, un sesgo dentro de la elección de estos puntos específicos (resultará obvia, por ejemplo, la predilección hacia una historia de la ciencia relatada desde una perspectiva occidental), pero se considera que esto tan solo hace referencia a la universalidad del tema que se busca tratar. No es más que un sueño inalcanzable el proponerse una exploración completa de cada punto que se podría prestar para un estudio de las creencias de una comunidad científica. Por el momento, habrá que conformarse con un recorrido que, aunque limitado, servirá de elemento esclarecedor dentro del entendimiento de los mecanismos con los que la maleabilidad de las creencias opera según el entorno. Dicho esto, se puede iniciar el recorrido por la historia de la ciencia, tomando como punto de inicio una civilización que, desde el Oriente Medio, sentó las bases de toda una cultura de pensamiento científico.

\section{LAS ETAPAS PRIMITIVAS DEL SER HUMANO}

En la transición de un modo de vida nómada a uno basado en asentamientos humanos es posible reconocer los primeros indicios de una ciencia (Liebenberg, 2013). En este sentido, a través de la observación, la humanidad habría logrado aprender sobre técnicas de agricultura y, a su vez, sobre los beneficios que supone mantener animales en cautiverio. Este fenómeno se extendió a lo largo de las ciudades de la Antigüedad (aunque ciertas urbes en específico destacaron en este aspecto).

En Sumeria, región del Oriente Medio, la antigua civilización construyó ciudades dedicadas a la innovación. Los sumerios desarrollaron un sistema de distribución del trabajo que aumentó su productividad. Este procedimiento significó que, mientras algunos individuos se encargaban de las tareas más agotadoras físicamente, otros tenían la posibilidad de dedicarse a empresas de un carácter mucho más abstracto. De esta manera, los sumerios realizaron aportes invaluables a la humanidad: desarrollaron el concepto de la rueda, estudiaron los cuerpos celestes y pensaron sobre arte, números y política (Kramer, 2010). A pesar de estos grandes avances científicos, los sumerios desarrollaron, al igual que muchas de las otras civilizaciones antiguas, un sistema de creencias religiosas. La observación de la bóveda celeste y el descubrimiento de las estaciones marcadas por ciclos lunares los llevó a concebir, incluso, una deidad lunar a la que nombraron Nanna (Hakim, 2004). Esto último tuvo que relacionarse con el hecho de que, en este punto de la historia, la ciencia aún no habría alcanzado los conocimientos necesarios para brindar explicaciones lógicas acerca de la mayoría de los fenómenos naturales. 
De esa manera, la civilización sumeria caracteriza un periodo de la historia en el que, en ausencia de una ciencia lo suficientemente madura como para brindar explicaciones de los fenómenos que presenciaban los antiguos pobladores de la Tierra, los seres humanos recurrieron a la producción de mitos para llenar aquel vacío conceptual que experimentaron. Tratar de justificar el dogma científico en esta etapa de la historia humana hubiese sido imposible, pues la ciencia aún era un campo reciente. No existía siquiera el concepto de continuidad de la empresa científica, elemento base del dogma, y, mucho menos, razones para confiar en este. Así, la comunidad científica de la Antigüedad (aquel grupo de personas dedicadas a asentar las bases de una ciencia futura, aun cuando lo hicieran sin saberlo) fue dominada, casi exclusivamente, por la creencia en elementos arquetípicos de carácter religioso.

\section{Jonia, IsaAC NeWTon y EL DEMONio de LAPLACE}

Dejando de lado a la civilización sumeria hay que transportarse unos cuantos miles de años hasta el siguiente punto de interés. De esta manera, Hakim (2004) reconoce en la civilización griega el inicio definitivo de una era de formalismo científico y explica de qué manera, en la región de Jonia, los griegos de la Antigüedad encontraron un lugar desprovisto de reyes que les concedió la libertad necesaria para prosperar en el comercio y, de manera simultánea, explorar terrenos desconocidos por el pensamiento humano. La ciencia floreció bajo el cuidado de Tales, primer filósofo, científico y matemático. Se dice que predijo un eclipse solar ocurrido en el año 585 a. C. (Couprie, 2011), desarrolló un sistema basado en triángulos para calcular la distancia de barcos con respecto a la costa (Panchenko, 1993) y descubrió el fenómeno de la electricidad al frotar una pieza de ámbar con lana (Iversen y Lacks, 2012). Mientras que los sumerios y los egipcios habían afirmado que el océano era la fuente de toda la vida, Tales buscó pruebas tangibles de esto. Junto a Tales, los pobladores de Jonia marcaron la transición de un mundo de mitologías y envuelto en misticismos hacia uno comprensible por el intelecto humano a través de su observación minuciosa.

Bajo esta perspectiva, Jonia no solo se alza como una cuna de la ciencia, sino también como cuna del ateísmo. De esta manera, los primeros indicios de una alternativa científica para la fe religiosa aparecieron en esta región de la costa de Turquía. Tales no observó el recorrido fantástico del titán Helios sobre su carruaje arrastrado por toros cuando presenció el movimiento del Sol sobre los cielos, sino un fenómeno totalmente predecible por leyes naturales. Esto conllevó tanto en Tales como en otros individuos el rechazo total de lo místico y lo inexplorable (Hakim, 2004). Otros pensadores retomaron la tarea de Tales y continuaron nutriendo las bases de una ciencia cada vez más cultivada, motivados ya fuera por las aplicaciones directas de esta última, ya por una atracción de un carácter mucho más abstracto, como la que cautivó a Tales.

No obstante, aún sería pronto en la historia de la ciencia para considerarla como una verdadera rival de la fuerza que ostentaban en esos tiempos los mitos y las explicaciones sobrenaturales o arquetípicas. De esta manera, pasarían siglos hasta que la ciencia se convirtiera en una opción digna de considerar, lo cual se conseguiría eventualmente al demostrar su capacidad de explicar y manipular el mundo natural, juez supremo de la validez de cualquier ciencia.

Ahora bien, el concepto del determinismo mecánico es otra de las tantas contribuciones de la civilización griega al pensamiento moderno. En este sentido, fueron Leucipo y Demócrito quienes concibieron la idea en el siglo $\mathrm{V}$ a. C. Ambos declararon que todos los fenómenos que se observan en el mundo son atribuibles al movimiento de los átomos indivisibles e indestructibles sobre un espacio de lo contrario vacío (Green, 1995). Esta concepción del mundo tomó particular fuerza con el surgimiento definitivo de la mecánica clásica. Se le llama así a la mecánica que obedece a las leyes formuladas por Newton en su libro Philosophiae Naturalis Principia Mathematica, o bien, a formulaciones equivalentes de estas, como sucede ser en el caso de la mecánica hamiltoniana (Hamilton, 1834) y lagrangiana (Lagrange, 1853).

A raíz de la introducción del cálculo y las leyes de Newton, se hizo posible indagar, con una nueva profundidad, la maquinaria oculta del universo. En su Principia, Newton sentó las bases de la mecánica 
celeste, el estudio del fenómeno gravitatorio y la descripción matemática de las ondas (Hakim, 2005); en un sentido más amplio, significó el inicio de una nueva era para la física, a la vez que la cuasi realización del sueño de Tales y cuantos le siguieron en su búsqueda de explicaciones sobre el mundo natural. Leucipo y Demócrito habían discernido siglos antes la idea de átomos interactuando entre sí, pero era Newton quien ahora parecía haber revelado las reglas de esta interacción.

Laplace intuyó las implicaciones de estos postulados cuando, en 1819, le dio vida al famoso demonio de Laplace. El matemático francés imaginó un demonio con conocimiento completo sobre las posiciones, velocidades y masas de todas las piezas de materia en el universo. Utilizando la mecánica newtoniana, el demonio sería capaz de predecir con exactitud infinita el estado del universo en cualquier tiempo pasado o futuro (Green, 1995). La cualidad demoníaca del personaje de Laplace se fundamenta entonces en la negación de cualquier forma de libre albedrío que este conlleva.

Esto no implica que nuestras decisiones no tengan impacto alguno en el curso de nuestras vidas, sino que estas ya se encuentran predefinidas desde el inicio de los tiempos. ¿Qué razón resta para la existencia misma?, ¿es la historia humana una película destinada a repetirse por toda la eternidad para el disfrute de un ser sobrenatural que no experimenta estas cadenas? Es natural que surja un sentimiento de desolación en quien encuentra por primera vez estas ideas. Ellas sugieren, a la vez, una nueva línea de preguntas o críticas hacia la noción de un diseño inteligente. Así, es común escuchar, dentro de discusiones teológicas, la justificación de que nuestra experiencia en el mundo terrenal es tan solo una preparación para lo que aguarda tras el umbral de la muerte. Holloway (2016) explica cómo, ante la imposición de un determinismo absoluto, esta idea se derrumba como un sinsentido, dado que la preparación a la que se refiere es tan solo una ilusión en la que los resultados ya son conocidos con anterioridad. ¿Con qué razón es, entonces, que se ha desarrollado en la mayoría de las religiones aquella típica narrativa sobre la lucha por la salvación de la humanidad? Para muchos, esta y otras preguntas de la misma especie admiten una sola respuesta: la negación absoluta de dios (cualquiera que sea la forma de interpretar a este en el contexto de cada religión en particular).

Green (1995) comenta que Laplace sostuvo, durante su vida, un ateísmo basado en una visión absolutamente determinista del mundo. Y Laplace no fue el único de las personas intelectuales de su época en declararse a sí mismo ateo. El siglo XVIII trajo consigo un cambio en la forma de observar el mundo, razón por la cual hoy se le denomina Siglo de las Luces. La comunidad intelectual de este siglo fue motivada por una visión de mundo basada en la ciencia y el raciocinio (Hakim, 2005). En síntesis, este fue un periodo de transición conceptual de un universo regido por la divina providencia a uno gobernado por leyes deterministas. Se reconoce, entonces, en este periodo histórico, dentro de la comunidad científica el comienzo definitivo de un proceso de acercamiento al dogma científico. Este ya no representa una apuesta tan arriesgada, pues la ciencia ha demostrado ser capaz de explicar los principales fenómenos del mundo natural. A partir de este punto, el ateísmo comenzó a arraigarse seriamente dentro de la comunidad científica, producto claro de las condiciones históricas y científicas de la época.

\section{LA REALIDAD EVANESCENTE DE LA MECÁNICA CUÁNTICA}

La acumulación de argumentos a favor del determinismo que sucedió a partir de Newton impactó severamente en la formación de las comunidades científicas posteriores. De esta manera, esto inculcó una creciente separación de todo elemento que pareciera pertenecer al reino de lo sobrenatural e inexplicable. No fue sino hasta el siglo XX que se encontró una prueba sólida, basada en principios científicos, en contra de la hipótesis determinista. A este periodo de la historia se le conoce como revolución cuántica. Dentro de este texto, servirá de escenario histórico para el desarrollo del tercer argumento a favor de la tesis sobre la maleabilidad de las creencias en función de las condiciones del entorno científico. La razón por la que se ha decidido tratar separadamente este tema, aun cuando también parece pertenecer al campo de la historia de la ciencia, será tratada explícitamente al final del ensayo. Se ha optado por esta forma de desarrollo, no con el fin 
de confundir al lector, sino porque se considera que, para el tratamiento de ciertos temas, es más beneficioso discutir primero un ejemplo y luego considerar el caso general.

Los inicios de la mecánica cuántica se encuentran en el campo de la termodinámica, específicamente, en el estudio de cuerpos negros. Newton (2007) reconoce a Lord Rayleigh como el primero en haber derivado una fórmula para la descripción de la radiación de un cuerpo negro a partir de la combinación de los principios termodinámicos y las leyes de Maxwell (Rayleigh, 1900). La incongruencia entre los resultados predichos por la teoría de Rayleigh y los observados experimentalmente permaneció como un misterio hasta que, en el año 1900, el físico Max Planck logró, a partir de una cuantización de la energía, hacer que ambos resultados concordaran (Planck, 1901). Para hallar una solución a su problema, Planck había sustituido un misterio por otro: ¿Por qué razón habría de cuantizarse la energía, cuyo continuo es asumido a lo largo de toda formulación clásica? Newton (2007) describe la forma en que el siguiente paso en la revolución cuántica surgió como consecuencia de otra situación extraña producida dentro del laboratorio: la incidencia de luz en un metal parecía expulsar, bajo ciertas condiciones, a los electrones de este metal (Lenard, 1902). El efecto fotoeléctrico - como es llamado actualmente este fenómeno recién descrito- no fue explicado sino hasta 1905 con la publicación de reveladores resultados por parte del físico Albert Einstein. Él, mediante dichos resultados, sugirió la cuantización de la energía transportada por un haz de luz (Einstein, 1905).

Otros acontecimientos contribuyeron con la puesta en marcha de la revolución cuántica, por ejemplo, el descubrimiento de los rayos X por Wilhelm Röntgen y el del fenómeno de la radioactividad por Henri Becquerel. Entre la serie de eventos que siguieron, cabe destacar la llegada del famoso modelo atómico de Bohr. En este, Bohr propuso que, entre todas las posibles órbitas que un electrón puede seguir alrededor del núcleo, solo unas cuantas eran físicamente alcanzables (Bohr, 1913). Eventualmente, Einstein realizó la conexión entre los resultados de Planck y Bohr y le dio nueva credibilidad a la idea de la cuantización (Einstein, 1906). El modelo de Bohr, sin embargo, no fue más que el último intento de parte de la física clásica por explicar el extraño comportamiento de los sistemas microscópicos. Al igual que sus modelos precursores, una serie de limitaciones en sus capacidades de coincidir con resultados experimentales, eventualmente, le ganaron a este la denominación de modelo obsoleto.

La incapacidad de la física clásica para explicar el comportamiento de los sistemas microscópicos ya comenzaba a hacerse notar y era evidente la necesidad de una nueva teoría para continuar con el desarrollo de la ciencia a escalas cada vez más pequeñas. Entonces, una vez comprendida la problemática que la mecánica cuántica moderna vino a solventar, se analizarán las características de esta teoría.

Newton (2007) reconoce como piezas fundamentales en el desarrollo de la mecánica cuántica moderna a las figuras de Heisenberg, Schrödinger y Dirac. Cada uno realizó aportes invaluables dentro de la construcción de un formalismo cuántico que estuviera en capacidad de realizar predicciones sobre sistemas físicos microscópicos. En este sentido, Heisenberg desarrolló su mecánica matricial expresada en el lenguaje del álgebra matricial (Heisenberg, 1925). Por su parte, Schrödinger desarrolló su mecánica ondulatoria expresada en el lenguaje de ecuaciones diferenciales (Schrödinger, 1926). Dirac comprobó que ambas mecánicas eran equivalentes, a la vez que generalizó el tratamiento al caso relativista (Dirac, 1926). No obstante, el desarrollo de toda teoría requiere no solo de su construcción, sino también de su comprensión. ¿Cómo era posible interpretar físicamente los resultados de la mecánica cuántica? Para responder esta pregunta es necesario recordar, quizá, la característica más crucial de la teoría: la mecánica cuántica es, en la mayoría de los casos, incapaz de describir la evolución temporal de propiedades físicas (a diferencia de la mecánica clásica). Se basa más bien en describir la evolución temporal de las densidades de probabilidad asociadas con cada una de las propiedades físicas medibles de un sistema en particular. La información sobre el estado físico de un sistema tan solo puede ser accedida mediante un acto de medición, el cual colapsa la densidad de probabilidad en el valor medido (Born, 1927).

Fue con esta pieza fundamental de información, acompañada de largas horas de discusión, que Bohr y Heisenberg (con contribuciones de Dirac y Schrödinger) le dieron vida a la hoy famosa interpretación de 
Copenhague (Bohr, 1937). La esencia de esta, que ya se ha ido comentando sin saberlo, se ve contenida en la siguiente frase: nada es real hasta que haya sido medido (Newton, 2007). Esta frase sirve para exacerbar el sentido probabilista de la teoría: previo a cualquier medición, el sistema y sus propiedades físicas existen en un estado de incertidumbre completamente incompatible con las ideas de determinismo que había cultivado la física clásica por años. Es de suma importancia mencionar que esta interpretación de Copenhague no cuenta con el apoyo unánime de toda la comunidad científica. Prueba de esto ha sido el surgimiento de múltiples interpretaciones alternativas (Collins, 2007), dentro de las cuales se podrían mencionar la interpretación de muchos mundos (IMM) o la interpretación de Bohm. La mecánica cuántica constituye así un misterio que sigue eludiendo hasta el día de hoy a las grandes mentes del siglo XXI. La lógica por la que opera aún esconde muchos de sus secretos y el hecho de que sea un área tan desacoplada de la experiencia cotidiana del ser humano tampoco ha ayudado en su proceso de comprensión.

\section{UNA NUEVA PERSPECTIVA}

Ante la falta de una interpretación satisfactoria de la mecánica cuántica, un creciente número de personas de ciencia han buscado nuevas interpretaciones fundamentalmente distintas a las que se mencionaron en la sección anterior. Ya se indicó cómo dentro del mundo cuántico nada es real hasta haber sido medido. Esta declaración ha sido, justamente, la que ha abierto las puertas a discusiones sobre el papel del observador dentro de un sistema físico. De esta manera, se muestra un particular interés en el impacto de su consciencia sobre la evolución del sistema. Así, los siglos XX y XXI han sido testigos del regreso de una tendencia hacia el misticismo dentro de la comunidad científica estudiosa de la mecánica cuántica.

A pesar de ello, quienes se han adentrado en esta propuesta del regreso hacia el misticismo no lo han hecho sin repercusiones. Como habrá adivinado la persona lectora, aquellas personas que se deciden por esta senda son culpadas de haber abandonado la empresa científica. A pesar del riesgo que supone esta postura, a medida que pasa el tiempo y el enigma cuántico continúa sin solución, el grupo de individuos que sostiene la propuesta del misticismo continúa creciendo en número y aumentando su popularidad (aquí se quiere dejar claro que, de ninguna forma, constituyen una mayoría, pero sí una porción lo suficientemente grande como para ser advertida). Si el fenómeno no ha sido notado en la mayoría de los campos de la ciencia, es porque el enigma cuántico no ha representado un obstáculo para la mayoría de las personas investigadoras. La ausencia de una interpretación satisfactoria no ha detenido el avance tecnológico que, en las últimas décadas, se ha basado en principios cuánticos (Yariv, 2013). Es posible resumir el enigma cuántico de tal manera que se ponga en evidencia este fenómeno: se sabe bastante sobre bajo qué mecanismos funcionan los sistemas microscópicos, pero poco acerca de las razones por las que suceden estos mecanismos. Lo anterior es un punto central y lo que ha motivado una serie de incursiones en busca de respuestas dentro de los territorios de lo místico (sobrenatural o arquetípico) de parte de algunos individuos. Convencidos de que la ciencia moderna es incapaz de responder sus preguntas, han optado por recurrir a explicaciones esencialmente metafísicas (no sorprende, entonces, que la mayoría haya tachado a este movimiento como una pseudociencia).

Una de las corrientes más influyentes en el proceso de formación de este grupo ha sido la del orientalismo, el cual basa el núcleo de sus creencias en las enseñanzas provenientes de sistemas filosóficos (y muchas veces religiosos) como el hinduismo, el budismo y el taoísmo (Capra, 2010). Como común denominador, es posible identificar en el pensamiento de estos individuos la búsqueda de una conexión mucho más íntima con lo que han llamado la conciencia universal: una extrapolación de la conciencia humana individual que permite establecer la idea de que todo el universo se encuentra conectado. Esta no es una idea nueva en lo absoluto. De hecho, es posible rastrear indicios de ella hasta miles de años atrás en la historia humana. Se podría hacer referencia, por ejemplo, al texto fundador del taoísmo, el Tao Te Ching, en el cual Lao-Tse escribió como primera oración que el Tao que puede ser expresado no es el Tao Absoluto, y los nombres que pueden decirse no son los Nombres Absolutos (Minford, 2018), insinuando así la existencia de una realidad subyacente a 
aquella que el mundo natural ofrece al manifestarse a los sentidos. Esta realidad subyacente podría, en última instancia, llegar a interpretarse como el espacio donde se desarrolla, en un estado oculto, tal consciencia universal. Así, las creencias de este grupo se han fundamentado en la adopción de doctrinas desarrolladas hace miles de años.

La formación científica de este grupo conlleva, sin embargo, una clara diferenciación entre ellos y los sabios que una vez estudiaron estas mismas doctrinas. Si estas creencias sobrenaturales o arquetípicas han de explicar el fenómeno cuántico, también han de tener un impacto directo en el mundo físico, más allá del mundo abstracto de las ideas al cual, tradicionalmente, se han limitado. De esta forma, han surgido, en las últimas décadas, estudios científicos que correlacionan aspectos espirituales con fenómenos mensurables (ver Colleso, 2017; Tiller et al., 1999). La veracidad y replicabilidad de estos estudios no son temas que se pretendan tratar en este ensayo. El aspecto que interesa dentro de esta discusión es que estos estudios han iniciado un proceso de reemplazo de creencias dentro de las comunidades científicas más cercanas al enigma cuántico. Así, las creencias de aquellas personas que forman parte de estos grupos han sido modificadas, lo que obedece, igual que en ocasiones anteriores, a las condiciones propias de su posicionamiento histórico.

Cabe mencionar que no constituye un fenómeno inédito en la historia de la humanidad el hecho de que haya surgido un grupo decidido a alejarse de las típicas corrientes científicas. Kuhn (2012), en su tratado sobre revoluciones científicas, explica cómo un elemento necesario para el surgimiento de cualquier revolución científica es un periodo previo de crisis. A medida que la ciencia avanza siguiendo cierto paradigma o forma de pensar, se encuentran anomalías que actúan como una piedra en el camino y, si permanecen mucho tiempo sin solucionarse, comienzan a sembrar dudas sobre la consistencia y utilidad del paradigma actual. Indudablemente, saldrán a la luz entonces una serie de individuos que, incapaces de soportar el nivel de tensión que involucra un periodo de crisis o incertidumbre en su campo, se embarcan en empresas científicas de carácter poco típico (que muchos, ni siquiera, llamarían ciencia). De esta forma, el tercer argumento, en términos generales, discute una dinámica útil para el estudio de cualquier periodo de crisis que haya sucedido o vaya a suceder dentro de la historia de la ciencia.

\section{Conclusiones}

A partir de la discusión de los tres argumentos, queda comprobado que las creencias dentro de la comunidad científica son fundamentalmente maleables. Al igual que ciertos materiales se deforman a través de una compresión o elongación, las creencias de los miembros de una comunidad científica pueden ser influenciadas $\mathrm{y}$, eventualmente, transformadas por las condiciones que se presenten en un periodo histórico en particular. Es así como, ante la falta de una ciencia madura, los mitos y las religiones prosperaron en las etapas tempranas del hombre en civilización (Hakim, 2004). Con el auge del determinismo científico, los individuos más cercanos a estos campos del conocimiento experimentaron una disminución de la necesidad de invocar elementos sobrenaturales o arquetípicos (Hakim, 2005). Actualmente, la confusión generada por la falta de una interpretación completa de la mecánica cuántica continúa alimentando a un movimiento de personas decididas a retornar a un misticismo olvidado, con la fe de que este esconda las respuestas que tanto se han buscado (Capra, 2010).

Así, se concluye que las condiciones que rodean a una comunidad científica en particular empujan a los miembros de esta comunidad hacia un acercamiento o alejamiento de lo que se ha llamado el dogma científico. Si de algo es testigo este hecho es de la variabilidad intrínseca en las creencias humanas, la cual actúa bajo el principio de la adaptación. La formación científica de una persona no la hace inmune a este fenómeno; incluso, parece exponerla a condiciones que inducen a una mayor variabilidad. La asimilación de las conclusiones de este ensayo constituye, entonces, una herramienta clave para comprender no solo a las comunidades científicas del pasado, sino también a las comunidades del presente y el futuro. A su vez, comprueba que no es posible nunca estudiar el fenómeno científico sin referirse a su aspecto humanístico. 


\section{Agradecimientos}

El autor corresponsal agradece la iniciativa de promover este tipo de investigaciones en el marco del curso FS-0741 Tópicos de Física IV (tema: historia de la física) de las carreras de Bachillerato en Física y Bachillerato y Licenciatura en Meteorología de la Universidad de Costa Rica (UCR). Adicionalmente, el coautor desea expresar su reconocimiento al apoyo de la Escuela de Física de la UCR por dar el espacio para impartir esta asignatura optativa, la cual pretende presentar la física desde el punto de vista de la historia de las ideas.

\section{REFERENCIAS BIBLIOGRÁFICAS}

Bohr, N. (1913). I. On the Constitution of Atoms and Molecules. The London, Edinburgh, and Dublin Philosophical Magazine and Journal of Science, 26(151), 1-25. doi: 10.1080/14786441308634955

Bohr, N. (1937). Causality and Complementarity. Philosophy of Science, 4(3), 289-298.

Born, M. (1927). Physical Aspects of Quantum Mechanics. Nature, 119(2992), 354-357. doi: 10.1038/119354a0

Capra, F. (2010). The Tao of Physics: An exploration of the Parallels between Modern Physics and Eastern Mysticism. Shambhala Publications.

Colleso, T. (2017). Direct Mental Influence on Random Event Generators [Undergraduate Honors Theses, King's University College]. Institutional Repository. https://ir.lib.uwo.ca/psychK_uht/61/

Collins, G. (2007, 19 de noviembre). The Many Interpretations of Quantum Mechanics. Scientific American. https:// www.scientificamerican.com/article/the-many-interpretations-of-quantum-mechanics/

Couprie, D.L. (2011). How Thales Was Able to Predict the Solar Eclipse of 28 May 585 B.C. En Heaven and Earth in Ancient Greek Cosmology. Astrophysics and Space Science Library (pp. 51-62). Springer-Verlag. doi: 10.1007/978-1-4419-8116-5_3

Dirac, P. (1926). On the Theory of Quantum Mechanics. Proceedings of the Royal Society of London. Series A, Containing Papers of a Mathematical and Physical Character, 112(762), 661-677. doi: 10.1098/rspa.1926.0133

Einstein, A. (1905). Über einem die Erzeugung und Verwandlung des Lichtes betreffenden heuristischen Gesichtspunkt. Annalen der Physik, 322(6), 132-148. doi: 10.1002/andp.19053220607

Einstein, A. (1906). Zur Theorie der Lichterzeugung und Lichtabsorption. Annalen der Physik, 325(6), 199-206. doi: 10.1002/andp.19063250613

Farias, M. (2013). The Psychology of Atheism. En S. Bullivant y M. Ruse (Eds.), The Oxford Handbook of Atheism (pp. 468-482). Oxford University Press.

Farias, M., Newheiser, A., Kahane, G. y De Toledo, Z. (2013). Scientific Faith: Belief in Science Increases in the Face of Stress and Existential Anxiety. Journal of Experimental Social Psychology, 49(6), 1210-1213. doi: 10.1016/ j.jesp.2013.05.008

Gray, J. (2004). Heresies: Against Progress and Other Illusions. Granta.

Green, R. (1995). The Thwarting of Laplace's Demon. Arguments Against the Mechanistic World-View. Palgrave Macmillan.

Hakim, J. (2004). The Story of Science: Aristotle Leads the Way. Smithsonian Books.

Hakim, J. (2005). The Story of Science: Newton at the Center. Smithsonian Books.

Hamilton, W. R. (1834). XV. On a General Method in Dynamics; by which the study of the motions of all free systems of attracting or repelling points is reduced to the search and differentiation of one central relation, or characteristic function. Philosophical Transactions of the Royal Society of London, 124(1), 247-308. doi: 10.1098/ rstl.1834.0017

Heisenberg, W. (1925). Über quantentheoretische Umdeutung kinematischer und mechanischer Beziehungen. Zeitschrift für Physik, 33(1), 879-893. doi: 10.1007/BF01328377

Holloway, R. (2016). A Little History of Religion. Yale University Press. 
Br. Juan José Bermúdez Vargas, et al. Sobre la maleabilidad de las Creencias de la comunidad Cient...

Iversen, P. y Lacks, D. J. (2012). A Life of its Own: The Tenuous Connection between Thales of Miletus and the Study of Electrostatic Charging. Journal of Electrostatics, 70(3), 309-311. doi: 10.1016/j.elstat.2012.03.002

Jung, C. (1954). Von den Wurzeln des Bewusstseins: Studien über den Archetypus. Psychologische Abhandlungen.

Kramer, S. N. (2010). The Sumerians: Their History, Culture, and Character. University of Chicago Press.

Kuhn, T. (2012). The Structure of Scientific Revolutions. University of Chicago Press.

Lagrange, J. L. (1853). Mécanique Analytique. Mallet-Bachelier.

Lenard, P. (1902). Ueber die lichtelektrische Wirkung. Annalen der Physik, 313(5), 149-198. doi: 10.1002/ andp. 19023130510

Liebenberg, L. (2013). The Origin of Science. CyberTracker.

Minford, J. (2018). Tao Te Ching: The Essential Translation of the Ancient Chinese Book of the Tao. Viking.

Newton, R. (2007). From Clockwork to Crapshoot: A History of Physics. The Belknap Press of Harvard University Press.

Panchenko, D. (1993). Thales and the Origin of Theoretical Reasoning. Configurations, 1(3), 387-414. doi:10.1353/ con.1993.0024

Planck, M. (1901). Ueber das Gesetz der Energieverteilung im Normalspectrum. Annalen der Physik, 309(3), 553-563. doi: $10.1002 /$ andp. 19013090310

Rayleigh, L. (1900). LIII. Remarks Upon the Law of Complete Radiation. The London. Edinburgh, and Dublin Philosophical Magazine and Journal of Science, 49(301), 539-540. doi: 10.1080/14786440009463878

Schrödinger, E. (1926). Quantisierung als eigenwertproblem. Annalen der Physik, 384(4), 361-376. doi: 10.1002/ andp. 19263840404

Tiller, W. A., Dibble, W. E. y Kohane, M. J. (1999). Towards Objectifying Intention via Electronic Devices. Subtle Energies \& Energy Medicine Journal Archives, 8(2), 103-123.

Yariv, A. (2013). An Introduction to Theory and Applications of Quantum Mechanics. Courier Corporation. 